\title{
Multiscale simulation strategies and mesoscale modelling of gas and liquid flows
}

\author{
Marco KALWEIT, Dimitris DRIKAKIS* \\ Fluid Mechanics and Computational Sciences Group, \\ Department of Aerospace Sciences \\ Cranfield University, Cranfield, MK43 0AL \\ United Kingdom \\ *Corresponding author: d.drikakis@cranfield.ac.uk
}

\begin{abstract}
This paper presents a review of multiscale simulation strategies for the modelling of micro- and nanoscale flows. These have been developed in the last two decades in an attempt to bridge the application gap between molecular and continuum simulation methods preventing the simulation of many micro- and nanofluidic devices. The paper is focused on hybrid molecular-continuum methods and reviews different coupling strategies, including geometrical decomposition in conjunction with state- and flux coupling, pointwise coupling, the heterogeneous multiscale method and the equation free approach. The different applications of these methods are briefly discussed.
\end{abstract}

Keywords: hybrid molecular-continuum methods, mesoscale, nanoscale, microscale

\section{Introduction}

The functionality of micro- and nanoscale fluidic devices is significantly determined by surface and molecular effects, as well as noncontinuum behaviour. For that reason, simulations of such devices cannot be carried out by relying solely on continuum models, in particular the most commonly used fluid model, the Navier-Stokes (NS) equations, because these have been developed from the macroscopic point of view. The NS equations are generally invalid when either the condition for continuum or for local thermal equilibrium breaks down. The continuum condition basically requires all material properties, kinematic properties, e.g., velocity and acceleration, and thermodynamic properties, e.g., pressure and density, to be defined at every point in space and to vary continuously and smoothly, i.e., derivatives of all dependent variables to exist within a defined accuracy threshold (Nyugen et al., 2006; Drikakis and Kalweit, 2006). Additionally, the NS equations assume a nearly linear relation of stress to strain rate and of heat-flux to temperature gradient. This only holds if the flow is locally at each point not too far from a thermodynamic equilibrium, which is only satisfied when the relaxation time is small compared to the employed numerical time step. In general, the continuum condition is mostly satisfied and it is the requirement for thermodynamic equilibrium that breaks down first (Gad-el Hak, 2006).

Molecular methods, such as molecular dynamics (MD) or the direct simulation Monte Carlo method (DSMC), describe the world from the nanoscopic perspective and model the physics accurately at these scales. However, these methods are no real alternatives as a design tool for engineering nano- and microscale fluidic systems, because of the extreme computational costs that currently limits their application to systems containing a few million particles, at least for those who do not have access to massive parallel computing clusters. Another restriction of molecular methods is that the small time step (for MD generally in the range of $10^{-15} \mathrm{~s}$ ) prohibits sufficiently long simulation times. This is especially problematic for slow flow speeds (a range in which many micro- and nanoscale fluidic devices will operate), because such flows usually develop slowly, thus extensive averaging is required to achieve an acceptable signal to noise ratio of any measured 
thermodynamic quantity. One may argue that progress in computing power will overcome these problems and enable molecular methods to fully simulate nano- and even micro fluidic systems for engineering purposes. This is unlikely to happen in the foreseeable future, where increased computing speeds are expected to be achieved primarily by increasing the number of processing units on a single processor, and only secondarily by increased calculation speed of the processing units themselves. Considering that the scalability of molecular methods is such that it requires a minimum number of particles per processor (about a few thousand), it is clear that the computable simulation time will still be determined by how fast a single processing unit can perform the required number of time steps for a few thousand particles.

For those reasons, there is an effort to develop mesoscale and multiscale methods with the aim to provide simulation and design tools for nano- and microfluidic engineering (Kalweit and Drikakis, 2008). This paper focuses on hybrid-molecular continuum simulations methods. Nevertheless, some alternative mesoscale methods are also discussed in the next section.

\section{Mesoscale Methods}

There is a range of mesoscale methods that may be used for the simulation of certain types of meso- and nanoscale flows. They are mostly based on the Boltzmann transport equation and can broadly be classified into pseudo particle methods and higher order moment methods. Generally, the validity of the Boltzmann transport equation for a specific material, a gas or liquid, depends on the employed collision integral. Thus, any model derived from this equation can strictly only be applied within the validity envelope of the collision integral. In the case of the classical Boltzmann transport equation, the collision integral is based on the kinetic gas theory and thus assumes binary collisions, molecular chaos, and point mass molecules. Hence, it is strictly only valid for gases and not for liquids. Algorithms to solve the Boltzmann transport equation itself are very complicated to implement for general applications and are therefore restricted to geometrically simple cases (Gad-el Hak,
2006). Higher order moment methods can be derived from the Boltzmann equation. Unlike their lower order counterparts, the Euler equation (zeroth order) and NS equation (first order), these equations contain higher order terms to account for departures from equilibrium, i.e., non-equilibrium effects. They are also known as extended hydrodynamic equations (EHE) (Agarwal et al., 2002). The most often used EHE is the Burnett equation, which was originally employed for rarified gas flows in the upper atmosphere, but has also been applied for microscale gas flows [Lockerby et al., 2005]. However, solving EHE is challenging and involves significant numerical and computational difficulties that restrict the application of EHE models. Furthermore, the EHE fail to capture some important microscopic phenomena, such as the Knudsen layer (Lockerby et al., 2005). Pseudo particle methods include the dissipative particle dynamics (DPD) method (Groot et al., 1997), the direct simulation Monte Carlo (DSMC) method (Bird, 1998) and the lattice Boltzmann method (LBM) (Succi, 2001). DPD is a mesh-less method and basically a coarse grained MD simulation. The fundamental particles are beads that comprise a number of atoms. All forces between particles smaller than the bead are averaged out and only the resulting forces between the beads are modelled. Originally, DSMC has been developed for high-speed rarified gas flows. It is not as efficient for the simulation of gas flows at micro- and nanoscale, because it suffers from slow convergence and large statistical noise under these conditions (Gad-el Hak, 2006). Most importantly, the DSMC performs best for rarified gas flows, while it is computationally expensive for simulation of gas under atmospheric pressure and cannot be used for dense gases and liquids. The LBM has a relatively simple algorithm that moves pseudo particles along a lattice. Two particles experience a collision when moving on the same lattice point. The method has been applied for rarified and continuum gas flows. It has also been used in the incompressible limit and for liquid flow. A limitation of the described mesoscale methods is that the 
description is based on average values that comprise the individual properties of a large number of atoms. That makes these methods unsuitable for any situation in which the behaviour can only be modelled appropriately by taking into account the interactions between individual atoms, such as it occurs in connection with more complicated biological molecules and on interfaces, where no accurate average description of the conditions is available. Typical examples are slip boundary conditions, for liquids (Squires, 2005).

Finally, another mesoscale approach recently proposed (Asproulis \& Drikakis, 2009) is based on neural networks, which can be used as an efficient tool to circumvent computer resource issues associated with molecular simulations and provide information on the effects of different sets of initial and boundary conditions.

\section{Hybrid molecular-continuum methods}

Besides the aforementioned mesoscale methods, multiscale simulation methods in the framework of hybrid molecular-continuum approaches have been developed to extend the application area of molecular and continuum method into mesoscales by providing a unifying description from nano to macro scales. Such methods have in common the coupling of a continuum solver, for fluids computational fluid dynamics (CFD), with a molecular solver, such as MD or DSMC (combinations with other mesoscale methods are also possible). In general, hybrid molecular-continuum methods (HMCM) work on the principle of decoupling length and/or timescales in order to minimise the application of the molecular solver spatially and temporally. The maximum number of particles that can be simulated is the most limiting factor and, therefore, all HMCM decouple the length scales by performing the full molecular simulation only within a single or multiple small sub domains of the entire simulation domain. The methods also decouple the timescale which reduces the length of the time period that has to be computed by the molecular solver. Decoupling of time scales is achieved by performing molecular simulations for selected periods only. In Figure 1 an overview of the current HMCM is presented. The classification is primarily made on how the length scales are decoupled, either by geometrical decomposition (GD) or point-wise coupling (PWC).



Figure 1: Hybrid molecular-continuum methods

\subsection{Geometrical decomposition}

For GD, as illustrated in Figure 2, the simulation domain is decomposed into a molecular and a continuum domain and the flow field is independently computed within each domain.

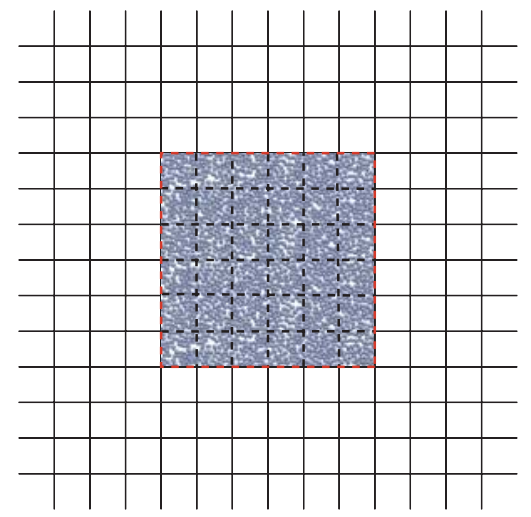

Figure 2: Geometrical decomposition

The molecular domain covers the part that cannot be modeled using continuum and must be small compared to the continuum domain for the approach to be efficient. A hybrid solution interface (HSI) between the subdomains establishes the connection between molecular and continuum to form one 
physical space. This is by no means an easy task and a number of different schemes have been proposed. In most cases it is realised by the continuum grid overlapping into the molecular domain. For fluidic applications, GD has been primarily applied to coupling of MD with CFD and DSMC with CFD.

On which type of quantity the coupling of MD and CFD should be based on has been a subject of some debate. Some researchers advocate coupling by state (Wijesinghe et al. 2004, Koumoutsakos, 2005), where the exchanged quantities are state variables, such as density, velocities or temperatures. The alternative approach, coupling by fluxes, preferred by others (Buscalioni et al. 2003, Flekkoy et al. 2005), is based on the exchange of mass, momentum and energy fluxes. Because of the difficulties in reconstructing microscopic states from macroscopic state variables, the state coupling approach is largely restricted to incompressible problems and was initially only applied to steady cases using a Schwartz alternating method to converge the molecular and continuum solutions. Only recently, the state coupling approach has been applied to unsteady cases. The flux coupling approach can be used for compressible unsteady flows as well and is suitable for scenarios, in which the characteristic time scales of the flow phenomena are close to molecular time scales, such as waves. No flux coupling method that also decouples time scales has been developed so far. Thus, these methods are limited in the computable time span by the fact that they are only spatial multiscale methods for which the computable time span is still dictated by the molecular simulation method (Wijesinghe et al., 2004).

Coupling of DSMC-CFD is basically state coupling and has been pioneered by Garcia et al. (1999) who suggested the AMAR (Adaptive Mesh and Algorithm Refinement) method that uses a Navier-Stokes solver in conjunction with mesh refinement as a natural framework for the introduction of the DSMC simulation at a certain refinement level. The first state coupling approaches for MD-CFD coupling were only applicable to scenarios without mass or energy transfer across the interface. O'Connel and Thompson (1995) simulated a one-dimensional incompressible Couette flow using a hybrid MD-CFD scheme for liquids based on an overlapping region between the CFD and MD domains for coupling of velocities using constrained Lagrangian dynamics. Hadjiconstantinou and Patera (1997) proposed the first twodimensional state coupling scheme. To enable mass flow across the MD-CFD interface, the rectangular $\mathrm{MD}$ region is embedded into a bigger simulation box with periodic boundary conditions serving as a particle reservoir. A Schwarz alternating scheme for the incompressible formulation was used as a time marching scheme to converge the solutions of the MD and CFD domains to a steady state. The method was first applied to Poiseuille and Couette flows of supercritical Argon and later to the moving contact line problem (Hadjiconstantinou 1999). The state coupling hybrid MD-CFD method was further developed by Werder et al. (2005). They disposed the reservoir region and used specular walls and consistent body forces to confine the atoms within the molecular region. Particle insertion and removal algorithms were used to ensure constant density in the boundary region and to realise in and out flow of mass. The scheme was demonstrated on the flow of a Lennard-Jones fluid past a carbon nanotube. Recently, Liu et al. (2008) extended the state coupling approach to unsteady flows. They demonstrated a channel flow with one oscillating wall that was covered by the molecular domain. The time scale decoupling has been achieved in a similar way as for the PWC, where short molecular simulations are performed for each macroscopic time step (Figure 4).

The pioneering work for Hybrid methods based on flux-coupling of incompressible flows was undertaken by Flekkoy (2001) and Wagner (2002). Their scheme coupled mass and momentum fluxes on the HSI. A reservoir region on the boundary of the $\mathrm{MD}$ region was 
used to implement the fluxes onto the molecular domain. Mass fluxes were implemented by the insertion or removal of atoms into or away from the reservoir region. The method was tested for incompressible Couette and Poiseuille flows. Buscalioni et al. (2003a) improved the flux coupling approach and extended it to compressible flows through incorporating the coupling of energy fluxes. This required the development of a search algorithm, referred to as USHER (Buscalioni et al. 2003), that seeks insertion sites for atoms and molecules at a desired energy level. The MD flux boundary conditions were verified for longitudinal and transversal waves in a onedimensional system and the fully coupled scheme was then tested on oscillatory wall flows (Buscalioni et al., 2004), though only for an incompressible isothermal fluid. The method was also applied to the simulation of a single tethered polymer in a solvent, subjected to oscillatory flow (Barsky et al. 2004). The USHER algorithm was extended by Fabritiis et al. (2004) to incorporate the insertion of molecules such as water. Improvements of flux boundary conditions for the MD domain were achieved by ensuring that the flux imposition fulfills the second law of thermodynamics, thus minimising the entropy production. Further work on flux coupling, in particular on MD flux boundary conditions was published by Kalweit et al. (2008, 2008a, 2008b).

The cell size of continuum grids that are used for flux coupling and other hybrid molecular-continuum methods that compute unsteady flow fields concurrently are for most cases small enough (at least near the HIS) to be subject to statistical fluctuations in the state variables. These fluctuations will occur naturally within the molecular domain, but must be introduced as additional terms into the continuum equation. This has been demonstrated by Fabritiis et al. (2006) for a MD-CFD flux coupling method, which was successfully tested on sound waves travelling in bulk water reflected by a lipid monolayer. Without the introduction of the fluctuation terms, the position of the HSI is clearly visible through the missing fluctuations in the continuum domain.

Previous reviews and discussions on the hybrid MD-CFD methods have been published by Wijesinghe et al. (2004), Koumoutsakos (2005) and Kalweit and Drikakis (2008).

\subsection{Point-wise coupling}

In contrast to the GD, where a part of the continuum domain is replaced by molecular modelling, in the point-wise coupling the continuum domain covers the entire simulation box. The molecular solver does not compute part of the flow field; it rather enters as a refinement (Ren et al., 2005). In the PWC one first defines a mesh to cover the domain of interest and solves the continuum equation for the nodes or cells, depending on the employed algorithm. The multiscale coupling is established by performing molecular simulations at the cell nodes to replace analytical and empirical models, where such are not available or cannot be formulated (Figure 3). In principle there are two types of relations:

A) Constitutive relations type problems and

B) boundary conditions type problems.

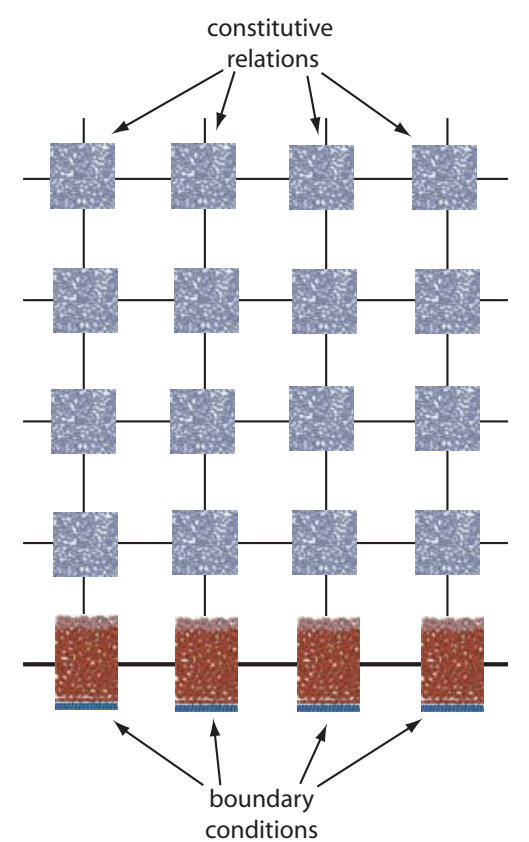

Figure 3: Point-wise coupling scheme 
Type A problems concern general cell nodes (or cell faces depending on the chosen macroscopic solver). For those, the molecular simulations are used to compute equations of state, such as the pressure, $p=p(\rho, c, T)$, or transport parameters, like the viscosity $\mu=\mu(\rho, c, T), \quad$ or diffusion coefficients $D=D(\rho, \boldsymbol{c}, T)$, for the current local macroscopic state, which may typically be presented by density $\rho$, species concentrations $\boldsymbol{c}$, and temperature $T$. In type $B$ problems, the molecular simulations are used to provide accurate boundary conditions such as tangential stress, $\tau_{\tau}=\tau_{\tau}(\rho, \boldsymbol{c}, T, u)$, heat flux, $q=q(\rho, \boldsymbol{c}, T, u)$ or slip velocity, $v_{s}=v_{s}(\rho, \boldsymbol{c}, T, u)$, for the current local macroscopic boundary state, represented typically by $\rho, \boldsymbol{c}, T$ and tangential flow speed on the boundary, $u$.

The length scale decoupling is achieved by keeping the molecular domains very small compared to the continuum cell size. Beside length scales, PWC also naturally decouples time scales by performing molecular simulations only for short time periods at every or selected continuum time steps. The timescale decoupling is also indicated in Figure 4, which shows that time periods simulated by the molecular solver are very small compared to the size of the continuum time step.
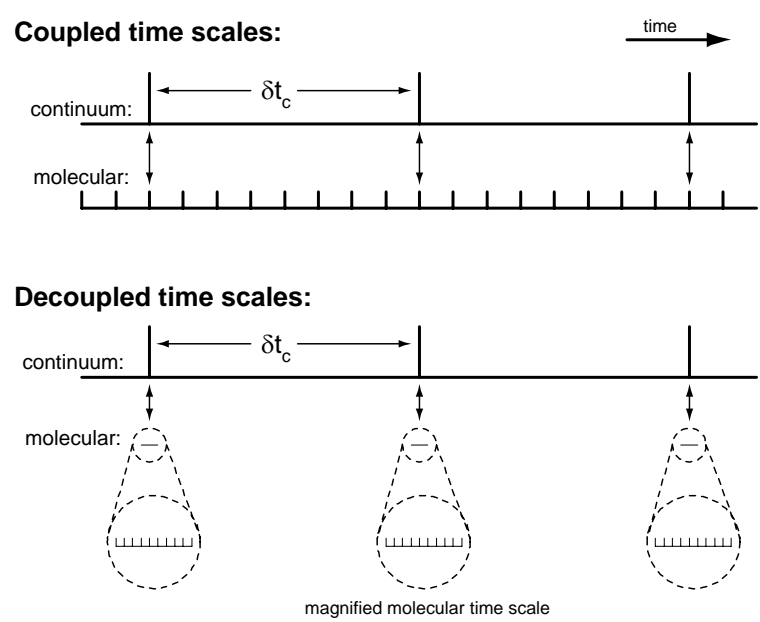

Figure 4: Coupled and decoupled time scales

In fact, the molecular solver performs a series of steady state simulations. Simulating the molecular system in a quasi-steady state presumes that the relaxation time of the molecular system, $\delta t_{r}$, (the time to reach a steady state and to obtain an accurate measurement) is small compared to the characteristic time scales of the simulated problem.

Several PWC type coupling methods for solid state and fluidic applications have been proposed in recent years under a variety of different names. However, they all work by the same principle, which has been described above. Kevrekidis and co-workers (2003) published the patch dynamics method, in which microscale simulations are performed at the grid nodes at regular macroscale time steps. The difference of the PWC is that no governing equations for continuum equation are employed to advance the solution between the macroscopic timestep. Instead, patch dynamics uses an interpolation algorithm. For that reason, it has been published together with the gap-tooth method under the common name "equation free approach" (Kevrekidis et al., 2003). The difference of the gap-tooth method is that it does not decouple the time scales, but simulates the patches (teeth) for the entire simulation time, like in GD (Gear et al., 2003). Ren et al. (2005, 2007) published the heterogeneous multiscale method and applied it successfully to pressure driven channel flows, cavity flows and contact line dynamics. A further improvement of the PWC type method is the storage and usage of molecular simulations already performed. This is achieved by using an algorithm that interpolates between results of molecular simulations that are carried out for discrete values of the state variable $\rho, c, T$ and $u$. This has been demonstrated for Poiseuille and shear flows of Lennard-Jones particles and polymers (Kalweit and Drikakis 2008, Asproulis and Drikakis 2009).

\section{Conclusions}

Several different hybrid molecularcontinuum methods for micro- and nano fluidic applications have been developed during the last two decades. There is no single 
method that covers all flow regimes; rather one has to choose a method depending on the nature of each specific case. PWC methods appears to cover the broadest application area as they are applicable for steady and unsteady flows when the characteristic time scales are large compared to the simulation time of the molecular solver. However, they cannot be applied in cases, such as waves generated by the fluid surface interactions, since these require a concurrent coupling, as provided through GD and flux coupling methods.

There is one regime that cannot be simulated by any hybrid multiscale method developed so far. This is when the molecular nature of the system changes slowly over macroscopic time scales. Typical examples are adsorption, sedimentation or fouling. Neither the long time scales over which the surface of a wall changes can be simulated by a molecular solver, nor can the dynamics of the build up be predicted by a macroscopic model that allows a re-initialisation of the molecular simulation. It remains to be seen whether such problems will be solved by hybrid molecularcontinuum or other mesoscale methods.

\section{References}

Agarwal, R. K. and Yun, K. Y.: 2002, chapter Burnett Simulations of Flows in Microdevices, MEMS Introduction and Fundamentals, Taylor \& Francis,

Boca Raton, FL, USA, pp. 7-1 - 7-33.

Asproulis, N. and Drikakis, D.: 2009, Nanoscale Materials Modelling Using Neural Networks, J. Comput. Theo. Nano Sci., 6, 514-518.

Barsky, S., Delgado-Buscalioni, R. and Coveney, P. V.: 2004, Comparison of molecular dynamics with hybrid continuummolecular dynamics for a single tethered polymer in a solvent, J. Chem. Phys., 121, 2403-2411.
Bird, G. A.: 1998, Molecular gas dynamics and the direct simulation of gas flows. Clarendon Press, Oxford.

Delgado-Buscalioni, R. and Coveney, P. V.: 2003, Usher: An algorithm for particle insertion in dense fluids, J. Chem. Phys., 119, 978-987.

Delgado-Buscalioni, R. and Coveney, P. V.: 2003a, Continuum-particle hybrid coupling for mass, momentum and energy transfers, Phys. Rev. E, 67, 046704.

Delgado-Buscalioni, R. and Coveney, P.: 2004, Hybrid molecular-continuum fluid dynamics, Phil. Trans. R. Soc. Lond. A, 362, 1639-1654.

Delgado-Buscalioni, R., Flekkoy, E. G. and Coveney, P. V.: 2005, Fluctuations and continuity in particle-continuum hybrid simulations of unsteady flows based on flux exchange, Europhys. Lett., 69, 959-965.

De Fabritiis, G. , Delgado-Buscalioni, R. and Coveney, P. V.: 2004, Energy controlled insertion of polar molecules in dense fluids, $J$. Chem. Phys., 121, 12139-12142.

De Fabritiis, G., Serrano, M., DelgadoBuscalioni, R. and Coveney, P. V.: 2007, Fluctuating hydrodynamic modelling of fluids at the nanoscale, Phys. Rev. E, 75, 026307.

Drikakis, D., Kalweit, M., Computational Modelling of Flow and Mass Transport Processes in Nanotechnology, First Handbook in Theoretical and Computational Nanotechnology (eds. M. Rieth, W.Schommers), American Scientific Publishers, Chapter 11, 470-545, 2006.

Flekkoy, E. G., Wagner, G. and Feder, J.: 2001, Hybrid model for combined particle and continuum dynamics, Europhys. Lett., 52, 271-276.

Gad-el Hak, M.: 2006, chapter Flow Physics, MEMS Introduction and Fundamentals, 
Taylor \& Francis, Boca Raton, FL, USA, pp. 7-7 - 4-35.

Garcia, A. L., Bell, J. B., Crutchfield, W. Y. and Alder, B. J.: 1999, Adaptive mesh and algorithm refinement using direct simulation monte carlo. J. Comput. Phys., 54, 134-155.

Gear, C. W. and Li, J. and Kevrekidis, I. G., 2003, The gap-tooth method in particle simulations, Physics Letters A, 316, 190-195.

Groot, R. D. and Warren, P. B.: 1997, Dissipative particle dynamics: Bridging the gap between atomistic and mesoscopic simulation, J. Chem. Phys., 107, 4423-4435.

Hadjiconstantinou, N. and Patera, A. T.: 1997, Heterogeneous atomistic-continuum representations for dense fluid systems, Int. $J$. Mod. Phys. C, 8(4),967-976.

Hadjiconstantinou, N. G.: 1999, Hybrid atomistic-continuum formulations and the moving contact-line problem. J. Comput. Phys., 154, 245-265.

Hadjiconstantinou, N. G.: 2005, Discussion of recent developments in hybrid atomisticcontinuum methods for multiscale hydrodynamics. Bulletin of the Polish Academy of Sciences, 53, 335-342.

Hyman, J. M.: 2005, Patch dynamics for multiscale problems, Computing in Science and Engineering, 7(3), 47-53.

Kalweit, M. and Drikakis, D.: 2008, Multiscale Methods for Micro/Nano Flows and Materials, J. Comput. Theo. Nano Sci., 5, 1923-1938.

Kalweit, M., Asproulis, N. and Drikakis, D.: 2008a, Nanofluidic applications of hybrid molecular continuum methods, Green Chemistry and Engineering International Conference on Process Intensification and Nanotechnology, pp. 189-199.
Kalweit, M. and Drikakis, D.: 2008b, Coupling strategies for hybrid molecularcontinuum simulation methods, Proc. I MECH E Part C J. Mech. Eng. Sc., 222(5), 797-806.

Kevrekidis, I. G., Gear, C. W., Hyman, J. M., Kevrekidis, P. G., Runborg, O., Theodoropoulos, C.: 2003, Equation-free coarse grained multiscale computation: enabling microscopic simulators to perform systems-level tasks, Comm. In the Math. Sciences, 14, 715-762.

Koumoutsakos, P.: 2005, Multiscale flow simulations using particles. Annu. Rev. Fluid Mech., 37:457-487.

Lockerby, D. A. and Reese, J. M. and Gallis, M. A.: 2005, The usefulness of higher-order constitutive relations for describing the Knudsen layer, Physics of Fluids, 17, 100609.

Liu, J., Chen, S., Nie, X. and Robbins, O.: 2008, A continuum-atomistic multi-timescale algorithm for micro/nano flows, Comm. Comput. Phys., 4(5), 1279-1291.

Nyugen, N. T. and Werely, S. T.: 2006, Fundamentals and Applications of Microfluidics, Artech House Inc., Norwood, MA, USA.

O’Connel, S. T. and Thompson, P. A.: 1995, Molecular dynamics-continuum hybrid computations: A tool for studying complex fluid flows, Phys. Rev. E, 52, 5792.

Ren, W. and Weinan, E.: 2005, Heterogeneous multiscale method for the modeling of complex fluids and micro-fluidics, J. Comput. Phys. 204(1), 1-26.

Ren, W.: 2007, Analytical and numerical study of coupled atomistic-continuum methods for fluids, J. Comput. Phys. 227(2), 1353-1371.

Squires, T. M. and Quake, S. R.: 2005, Microfluidics: Fluid physics at the nanoliter scale, Reviews of Modern Physics, 77, 9771025. 
Succi, S.: 2001, The lattice Boltzmann equation for Fluid Dynamics and Beyond, Oxford University Press, Oxford, UK.

Wagner, G., Flekkoy, E., Feder, J. and Jossang, T.: 2002, Coupling molecular dynamics and continuum dynamics, Computer Physics Communications, 147, 670-673.

Werder, T. , Walther, J. and Koumoutsakos, P.: 2005, Hybrid atomistic-continuum method for the simulation of dense fluid flows, $J$.

Comput. Phys., 205, 373-390.

Wijesinghe, H. S. and Hadjiconstantinou, N. G.: 2004, Discussion of Hybrid AtomisticContinuum Methods for Multiscale Hydrodynamics, Int. J. Multiscale Comput. Eng., 2, 189-202. 